\title{
EchoGéo
}

40 | 2017

Ressources urbaines (2)

\section{Du Oaxaca à la ville de Mexico : l'accès aux ressources urbaines}

\section{Antonine Ribardière}

\section{(2) OpenEdition \\ 12 Journals}

Electronic version

URL: https://journals.openedition.org/echogeo/14961

DOI: 10.4000/echogeo.14961

ISSN: 1963-1197

\section{Publisher}

Pôle de recherche pour l'organisation et la diffusion de l'information géographique (CNRS UMR 8586)

\section{Electronic reference}

Antonine Ribardière, "Du Oaxaca à la ville de Mexico : l'accès aux ressources urbaines ", EchoGéo [Online], 40 | 2017, Online since 30 June 2017, connection on 11 August 2021. URL: http:// journals.openedition.org/echogeo/14961 ; DOl: https://doi.org/10.4000/echogeo.14961

This text was automatically generated on 11 August 2021

EchoGéo est mis à disposition selon les termes de la licence Creative Commons Attribution - Pas d'Utilisation Commerciale - Pas de Modification 4.0 International (CC BY-NC-ND) 


\title{
Du Oaxaca à la ville de Mexico : l'accès aux ressources urbaines
}

\author{
Antonine Ribardière
}

1 La notion de ressources urbaines articule plusieurs niveaux, à commencer par ceux relatifs aux lieux. À l'échelle locale, le quartier est caractérisé par un ensemble de ressources qui le valorise ou au contraire, par une carence de ressources qui s'impose à l'ensemble des habitants ; à l'échelle de l'agglomération, suivant sa taille et sa position dans la hiérarchie urbaine, la ville elle-même est porteuse de ressources. À cet égard, Mexico, capitale politique et économique incontestée du Mexique, occupe une position privilégiée. Les ressources de la capitale ne sont pourtant pas également accessibles à la population résidente : c'est bien en fonction des ressources dont chacun dispose que se dessine l'accès à la ville. Ces ressources se définissent au niveau individuel - ce sont les compétences de l'individu, ou encore son patrimoine - et également au niveau du réseau social et familial, comme intermédiaire voire comme multiplicateur des ressources de l'individu et de celles de son environnement.

2 La position de migrant constitue un point de départ privilégié pour saisir la manière dont se constituent les ressources urbaines, à la croisée entre ces différents niveaux. Dans un pays comme le Mexique, où les différentiels en termes d'emplois et de conditions de vie entre villes et campagnes continuent de nourrir une migration d'origine rurale, c'est bien le potentiel de la ville qui est à l'origine du projet migratoire. Les flux de type rural-urbain n'ont certes pas la même ampleur que lors des décennies de l'explosion urbaine, toutefois, la migration depuis les zones rurales vers les zones métropolitaines représente encore près de $11 \%$ du total des flux observés entre 2005 et 2010 à l'échelle du pays (Pérez et Santos, 2013) ${ }^{1}$.

3 Les modes d'insertion des migrants d'origine rurale dans la société urbaine ont fait l'objet d'une littérature importante, en particulier au Mexique. Au cours des années 1970, alors que le taux de croissance moyen annuel de la ville de Mexico est encore de $4,5 \%$ et que la question migratoire est intimement liée à celle de la pauvreté urbaine et de l'habitat des masses populaires, L. Lomnitz se saisit de la notion de marginalidad ${ }^{2}$ pour rendre compte des conditions de vie dans un quartier populaire auto-construit en 
périphérie de la ville (Lomnitz, 1975) ${ }^{3}$. À une autre échelle d'analyse, J. Turner inscrit ce type de peuplement dans un modèle de mobilité résidentielle des migrants, du centre vers la périphérie, en lien avec leur niveau d'intégration socio-économique (Turner, 1968). Progressivement, l'analyse des modes de production de l'habitat populaire s'autonomise relativement à celle des migrations paysannes (Connolly, 1981) et s'intègre dans une approche globale des formes de divisions socio-spatiales urbaines (Schteingart, 1989; 2002; Duhau et Giglia, 2008; Valette, 2016). En parallèle, les migrations d'origine rurale sont abordées suivant des perspectives complémentaires ; nous en retenons deux, particulièrement présentes dans le champ de la géographie contemporaine. La première tient à l'exploration des différentes formes de territorialités engendrées par les expériences migratoires, nationales et internationales (Baby-Collin et al., 2009; Quesnel, 2009 ; Michel et al., 2011 ; Cortes et Pesche, 2014); la deuxième intègre la question de l'insertion urbaine des migrants à l'analyse des mobilités urbaines au sens large, réunissant mobilités résidentielles et mobilités quotidiennes, dans l'optique d'une approche compréhensive des dynamiques de production et de changement urbain (Dureau et Lévy, 2007 ; Delaunay, 2010 ; Dureau et al., 2014). La démarche que nous suivons ici est moins englobante : il s'agit avant tout de participer à définir la notion de ressources urbaines, à l'interface entre trajectoires des lieux et trajectoires des individus et ce faisant, de contribuer à la formalisation d'outils conceptuels à même de rendre compte des processus de production et de reproduction des inégalités en milieu urbain.

4 Au cours d'une enquête de terrain menée en 2014 dans l'Isthme de Tehuantepec (Michel, Ribardière, 2016), nous avions pu identifier un lien privilégié entre un village de l'État de Oaxaca, Santo Domingo Petapa, et un municipe industriel à l'ouest de la zone métropolitaine de Mexico, Naucalpan. Dans ces terres pauvres ${ }^{4}$ et traditionnelles de départ, non seulement la migration vers Mexico nous avait semblé toujours vivace, mais également particulièrement concentrée : le recensement de 2010 indiquait en effet que près d'un départ sur deux avait pour destination le seul municipe de Naucalpan. Nous avons ainsi choisi de focaliser notre approche sur la migration vers Naucalpan et en provenance du Oaxaca, en mobilisant deux types de matériaux. Premièrement, un matériau empirique a été collecté en juillet 2015 lors d'un travail de terrain réalisé avec Aurélia Michel (Michel, 2017), principalement dans la colonie populaire ${ }^{5}$ de Loma Colorada où nous avons retrouvé, entre autres, des ressortissants de Santo Domingo Petapa. Au total, nous disposons ainsi de la trajectoire d'une vingtaine de familles de migrants d'origine rurale, essentiellement du Oaxaca et installées à Mexico depuis la fin des années 1990 pour le plus grand nombre 6 . Deuxièmement, ce matériau empirique est croisé avec une approche systématique des résidents du municipe de Naucalpan originaires du Oaxaca ${ }^{7}$, issue d'une exploitation de l'échantillon détaillé du recensement 2010 de l'INEGI - la muestra -, exploitation que nous avons conduite avec l'aide de Jean-François Valette ${ }^{8}$.

5 Avant d'aborder les modalités de l'insertion urbaine des migrants, il nous faut présenter rapidement le municipe de Naucalpan. Ce municipe constitue le contexte de l'étude, au sens le plus large du terme: il forme non seulement l'environnement à partir duquel les migrants accèdent à la ville, mais également, par les ressources qu'il offre, il autorise voire détermine en partie leurs trajectoires. Nous nous concentrerons ensuite sur l'accès à deux ressources élémentaires, l'emploi puis le logement. 


\section{Naucalpan : ressources du lieu}

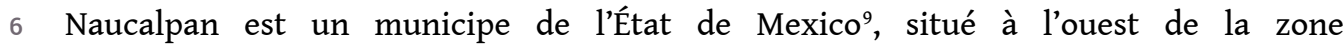
métropolitaine, bien relié au centre notamment par le métro (illustration 1): le terminus de la ligne 2 se situe en effet au niveau de la limite orientale du municipe, terminus à partir duquel les bus et microbus prennent le relais pour desservir l'ensemble de la zone. Le trafic est ainsi incessant entre le centre ancien de Naucalpan, San Bartolo et le terminus du métro, el Toreo (station Cuatro Caminos). Cette bonne accessibilité constitue une ressource certaine pour les habitants, elle autorise un approvisionnement au centre de la zone métropolitaine à moindre coût - que ce soit pour les besoins des ménages ou pour une éventuelle activité commerciale.

7 Le municipe compte en 2010 plus de 800000 habitants et s'étend sur près de 16000 hectares, toutefois seule la moitié est se trouve aujourd'hui urbanisée. Les colonies les plus occidentales se situent ainsi sur le front d'urbanisation de la zone métropolitaine, tandis que les colonies les plus anciennes, développées à partir de San Bartolo, sont aujourd'hui bien consolidées. C'est le troisième municipe le plus peuplé de l'État de Mexico, derrière Ecatepec et Netzahualcóyotl, à l'Est. Contrairement à Netzahualcóyotl, essentiellement peuplé sous la forme de colonies populaires, Naucalpan se caractérise par une grande hétérogénéité socio-spatiale.

Illustration 1 - Naucalpan : situation dans la zone métropolitaine de Mexico

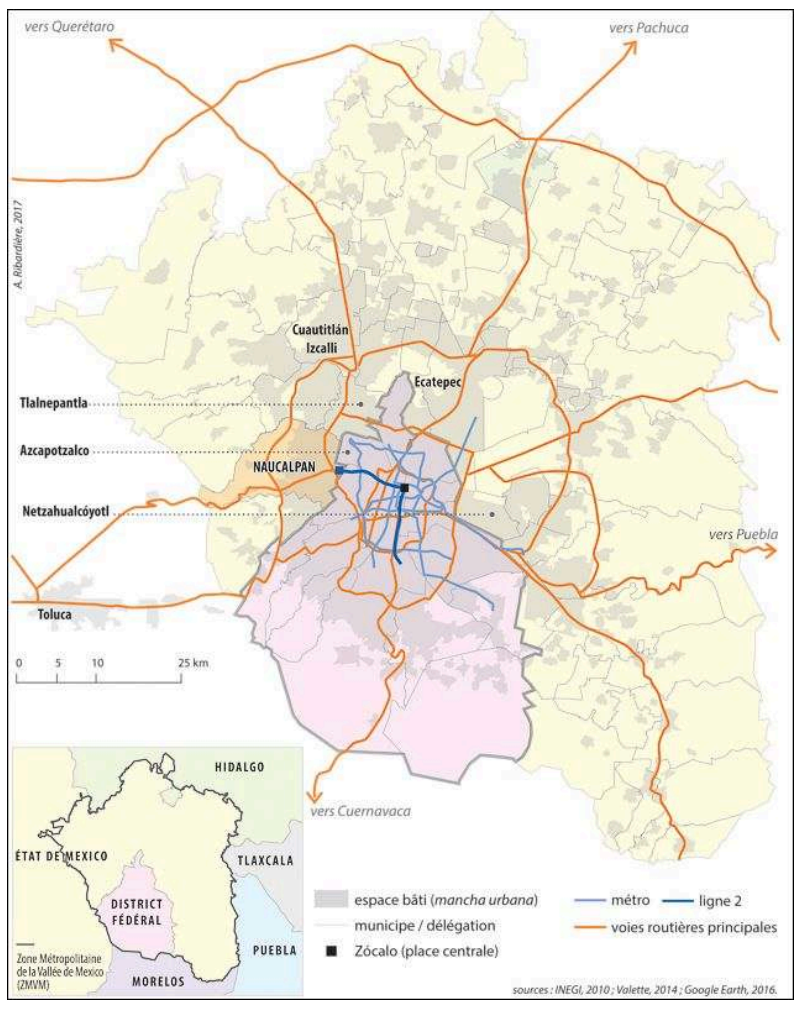




\section{La diversité socio-spatiale du municipe}

8 L'hétérogénéité sociale qui caractérise le municipe s'organise spatialement en quartiers bien distincts, en termes de densité, de forme de peuplement et de qualité des logements, de caractéristiques socio-économiques de la population résidente.

Nous illustrons ici cette diversité socio-spatiale en nous appuyant sur une typologie précédemment élaborée (Ribardière et Valette, 2014) qui décrit les formes sociospatiales de pauvreté à l'échelon des unités de recensement (illustration 2). En croisant plusieurs indicateurs d'ordre socio-économique ${ }^{10}$, cinq types d'unités spatiales ont ainsi pu être identifiés, depuis celui où la pauvreté est la plus marquée, jusqu'à celui qui apparaît le plus épargné par les formes de concentration spatiale de pauvreté.

Illustration 2 - L'hétérogénéité du tissu résidentiel : formes socio-spatiales de pauvreté dans la zone métropolitaine de Mexico

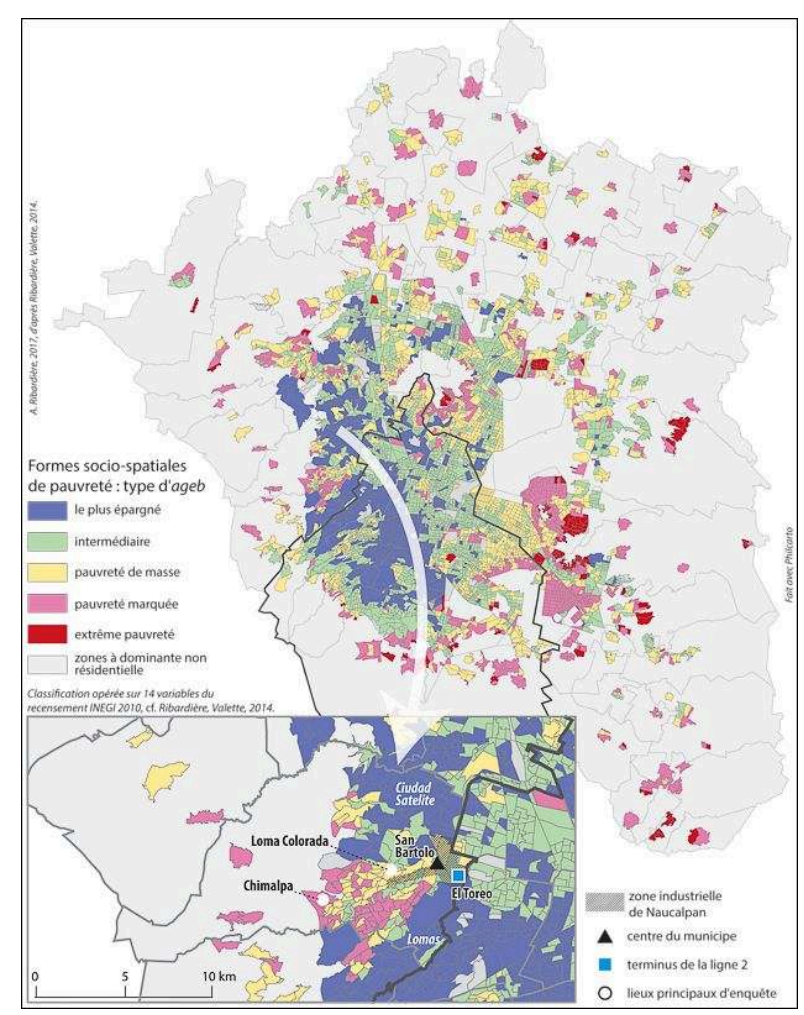

10 Au total, seul le type caractérisé par une surreprésentation des formes extrêmes de pauvreté n'est pas représenté à Naucalpan - tous les autres types le sont. On repère sur l'illustration 2 le centre traditionnel San Bartolo, les colonies populaires consolidées à proximité (dont Loma Colorada où s'est déroulé l'essentiel de l'enquête, cf. également illustration 3), les colonies populaires plus récentes, moins accessibles et aux formes d'habitat souvent plus précaires sur le front d'urbanisation à l'Ouest, caractérisées par une pauvreté marquée, et enfin, les zones résidentielles aisées. Ces dernières correspondent d'une part à la ville nouvelle de Ciudad Satellite au Nord, sortie de terre à la fin des années 1950, d'autre part aux zones résidentielles aisées dans le prolongement des Lomas de Chapultepec au Sud. Cette proximité relative des quartiers riches et des colonies pauvres mérite d'être soulignée, dans la mesure où elle peut 
représenter une ressource en terme d'emplois - nous le verrons au sujet des emplois domestiques.

Illustration 3 - Une rue passante dans la colonie populaire Loma Colorada, qui mène à l'école primaire Anahuac

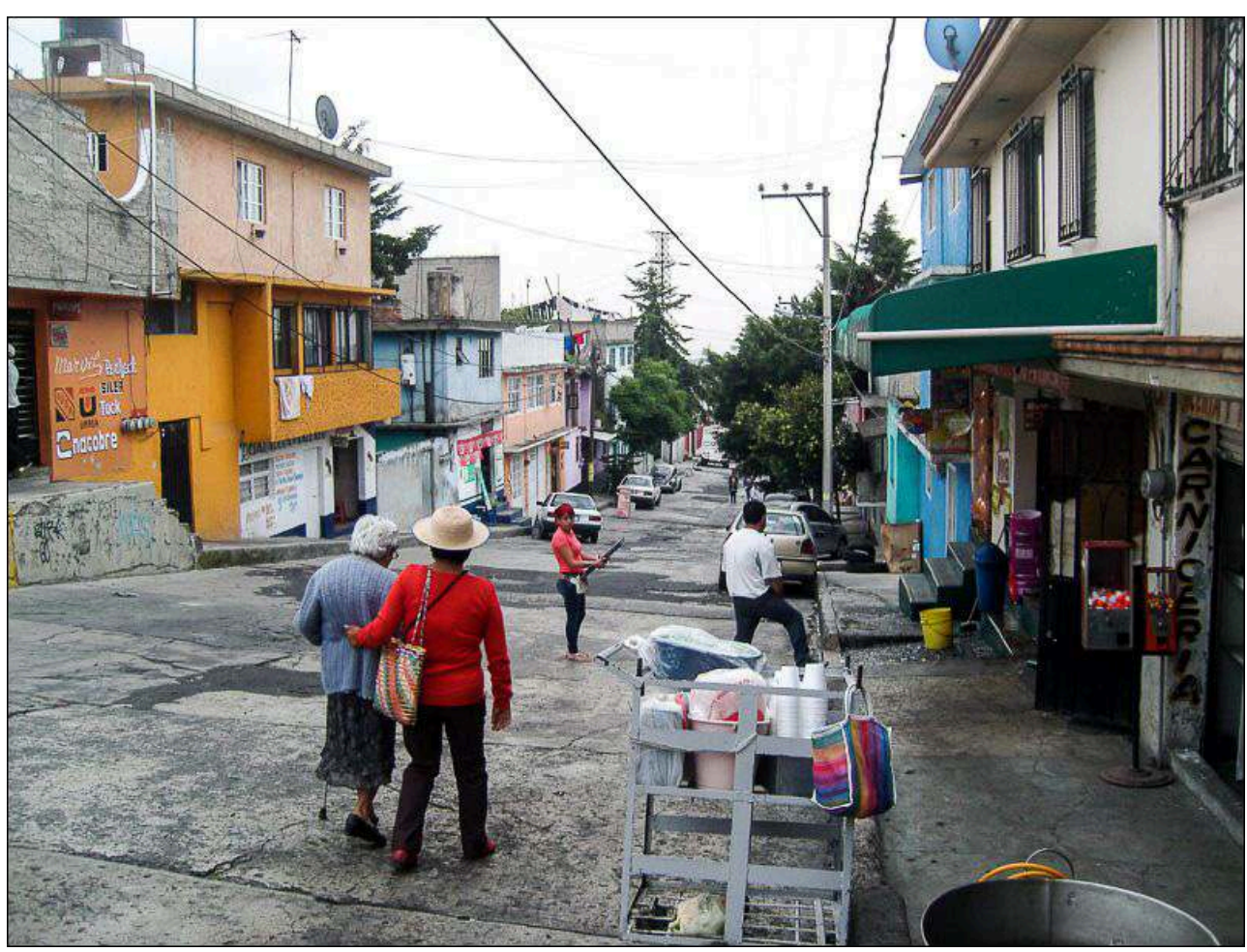

Auteur : A. Ribardière, juin 2015

\section{Les ressources locales en termes d'emplois}

La présence d'emplois dans le municipe ou dans les municipes voisins, en termes de service, mais également en termes d'emplois industriels, constitue une autre caractéristique importante relativement à notre problématique.

Naucalpan se situe dans le corridor industriel du Nord-Ouest de la capitale, qui s'est étendu depuis la délégation d'Azcapotzalco jusqu'à Cuautitlán Izcalli au Nord, à la faveur des premiers mouvements de déconcentration industrielle des années 1960. En 2013, les trois municipes ou délégations contigus de Tlalnepantla, Naucalpan et Azcapotzalco comptabilisent à eux seuls plus de 150000 emplois dans le secteur industriel, dont 46600 dans la seule zone industrielle de Naucalpan où dominent l'industrie chimique et pharmaceutique, la fabrication de produits plastiques (sacs, bouteilles et autres conditionnements) et de pièces métalliques (Inegi, Censos Económicos, 2014). Mexico reste aujourd'hui encore une agglomération industrielle et le municipe de Naucalpan se trouve ainsi particulièrement bien doté de ce point de vue, avec $26 \%$ des emplois dans le secteur en 2013. Services et commerce, selon la grande variété englobée dans ces catégories, représentent respectivement $37 \%$ et $31 \%$ des emplois.

13 Au total, selon la muestra de 2010 , ce sont plus des $2 / 3$ des personnes résidentes ${ }^{11}$ à Naucalpan qui travaillent dans le municipe : cette proportion est nettement supérieure 
à celle qui vaut en moyenne dans les municipes et délégations qui composent la ZMVM $(57,4 \%)$. Fait remarquable, la part des résidents travaillant dans le municipe augmente encore si on focalise l'observation sur les migrants récents en provenance du Oaxaca ${ }^{12}$ : elle concerne alors près de 9 personnes sur 10. Les lieux d'emplois des migrants oaxaqueños plus anciens apparaissent plus diffus, tout en restant tout de même concentrés sur Naucalpan pour 7 personnes sur 10. Autrement dit, des ressources locales existent à l'échelle du municipe et elles apparaissent largement accessibles aux migrants en provenance des États ruraux du Sud du pays, tel que le Oaxaca.

\section{Un municipe qui continue d'être attractif pour les migrants}

La croissance de Naucalpan rend compte de celle de l'agglomération, en termes de rythme, d'étalement urbain et d'apports migratoires dans le peuplement. L'explosion démographique observée entre 1960 et 1970 - le poids démographique du municipe se trouve alors multiplié par 4,7 - témoigne du rôle des municipes conurbés de l'État de Mexico dans la croissance de l'agglomération. Le cas de Naucalpan n'est pas isolé : le municipe voisin de Tlalnepantla connaît une augmentation du même ordre, tandis qu'à l'Est, le municipe de Netzahualcóyotl voit sa population multipliée par 10 au cours de la même période.

Illustration 4 - Croissance démographique de la ZMVM et du municipe de Naucalpan

\begin{tabular}{|l|l|l|}
\hline & $\begin{array}{l}\text { Population de Mexico (ZMVM), en milliers } \\
\text { d'hab. }\end{array}$ & $\begin{array}{l}\text { Population du Municipe de Naucalpan, en } \\
\text { milliers d'hab. }\end{array}$ \\
\hline 1950 & 2982,1 & 21,8 \\
\hline 1960 & 5155,3 & 92,0 \\
\hline 1970 & 8856,8 & 429,0 \\
\hline 1980 & 13734,6 & 730,2 \\
\hline 1990 & 15563,8 & 786,5 \\
\hline 2000 & 18396,7 & 858,7 \\
\hline 2010 & 20116,8 & 833,8 \\
\hline
\end{tabular}

Sources : recensements de population de l'INEGI ;

15 L'apport migratoire en provenance des campagnes est considérable au cours des années 1960 et 1970 ; à partir des années 1980 la migration du rural vers l'urbain diminue, en stock, mais surtout en poids relatif : ce sont les migrations intra-urbaines qui prennent alors de l'ampleur et assurent l'essentiel des recompositions de peuplement au sein de la zone métropolitaine (Valette, 2014). L'analyse du rôle de la migration dans la dynamique démographique de Naucalpan d'une part et des flux en provenance du Oaxaca vers la ZMVM d'autre part, s'inscrit complètement dans cette tendance métropolitaine. 
16 L'échantillon détaillé du recensement de la population mexicaine permet d'aborder la migration à travers deux entrées : la population enquêtée dans la muestra (soit environ $10 \%$ de la population totale) est interrogée sur son État de naissance et sur son municipe de résidence 5 ans avant la date du recensement. En conséquence, la migration récente peut être observée à l'échelon du municipe; en revanche la migration plus ancienne ${ }^{13}$ ne peut être considérée qu'à l'échelon de l'État. À travers ces données, on prend la mesure du rôle de la migration dans le peuplement de Naucalpan : en 2010 , plus de $48 \%$ de la population résidente dans le municipe est née en dehors de l'État de Mexico. Toutefois, plus de la moitié de ces migrations correspondent à des migrations de proximité, depuis le DF (District Fédéral) voisin, et renvoie au processus d'étalement urbain signalé plus haut. Pour le reste, le Oaxaca apparaît parmi les premiers foyers d'origine des migrants hors zone centrale (illustration 5) : au total, $6 \%$ de la population résidente à Naucalpan est née dans le Oaxaca.

Illustration 5 - État de naissance de la population résidente dans le municipe de Naucalpan en 2010 - hors État de Mexico (10 premières origines)

\begin{tabular}{|l|l|}
\hline État de naissance - hors État deMexico (10 premières origines) & Population, en nombre d'habitants \\
\hline District Federal & $\mathbf{2 2 4 0 5 4}$ \\
\hline Veracruz & 25091 \\
\hline Michoacan & 24188 \\
\hline Oaxaca & $\mathbf{2 3 6 7 7}$ \\
\hline Puebla & 20200 \\
\hline Hidalgo & 17302 \\
\hline Guanajuato & 11770 \\
\hline Guerrero & 10777 \\
\hline Jalisco & 6503 \\
\hline Chiapas & 5382 \\
\hline
\end{tabular}

Sources : Inegi, Muestra, 2010.

Illustration 6 -Origine de la population résidente dans le municipe de Naucalpan en 2010, résidant dans un autre municipe en 2005 ( 10 premières origines + État de Mexico)

\begin{tabular}{|l|l|}
\hline $\begin{array}{l}\text { Origine des résidents dans un autre municipe en 2005 } \\
(10 \text { premières origines + État de Mexico) }\end{array}$ & $\begin{array}{l}\text { Population de 5 ans et plus, en } \\
\text { nombre d'habitants })\end{array}$ \\
\hline État de Mexico & 19653 \\
\hline District Federal & 13655 \\
\hline
\end{tabular}




\begin{tabular}{|l|l|}
\hline Veracruz & 2959 \\
\hline Oaxaca & 2541 \\
\hline Guanajuato & 1984 \\
\hline Guerrero & 1369 \\
\hline Baja California & 1138 \\
\hline Puebla & 1106 \\
\hline Hidalgo & 1073 \\
\hline Sonora & 1044 \\
\hline Chiapas & 1038 \\
\hline
\end{tabular}

Sources : Inegi, Muestra, 2010.

La migration récente concerne une part de la population plus restreinte : un peu moins de $7 \%$ de la population recensée en 2010 à Naucalpan résidait dans un autre municipe en 2005 (illustration 6). Les flux observés témoignent de l'ampleur des migrations intramétropolitaines contemporaines: près de $60 \%$ des arrivées récentes à Naucalpan proviennent soit de municipes de l'État de Mexico, soit du DF. Les flux d'origine plus lointaine ne sont pourtant pas complètement taris et le Oaxaca constitue le deuxième État de provenance hors zone métropolitaine en 2010. En outre, le rôle de Naucalpan comme lieu d'accueil de la migration oaxaqueña se renforce: si la migration ancienne apparaît davantage ancrée à l'Est de la métropole, dans la délégation d'Iztapalapa et dans le municipe de Netzahualcóyotl en particulier, Naucalpan constitue en 2010 le deuxième point d'ancrage de la migration récente en provenance du Oaxaca (illustration 7).

Illustration 7 - Les migrants originaires du Oaxaca dans la zone métropolitaine de Mexico

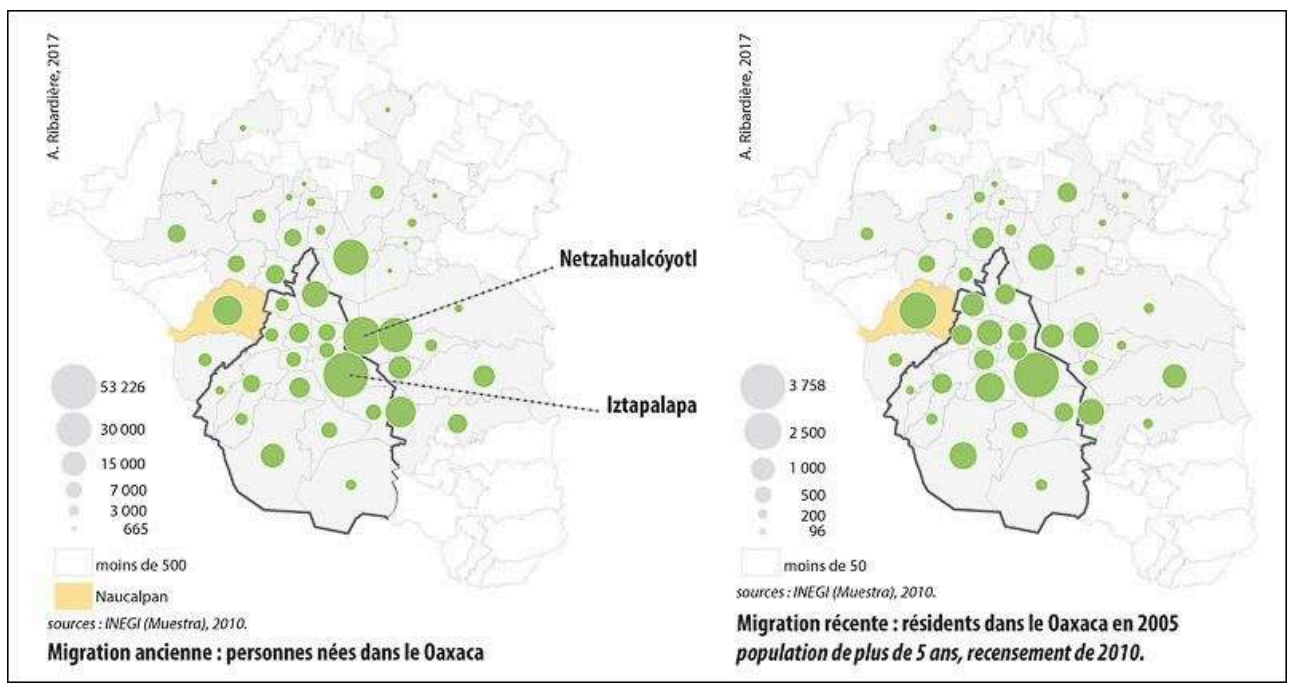


18 La ville de Mexico continue donc d'être une destination pour les migrations rurales même si, d'une part, la ville est traversée par des flux migratoires internes de plus grande ampleur qui sont aujourd'hui les principaux moteurs de l'étalement urbain et d'autre part, d'autres destinations de migration sont possibles : vers le nord, vers les zones touristiques, ou vers les États-Unis (Viramontes et al., 2013). Si les délégations centrales du DF (Cuauhtémoc, Gustavo A. Madero, Benito Juárez) continuent de recevoir les migrations extra-métropolitaines, les délégations périphériques (Iztapalapa) et les municipes péri-centraux de l'État de Mexico, aux premiers rangs desquels Ecatepec et Naucalpan, constituent aujourd'hui des lieux majeurs d'accueil des migrants ${ }^{14}$. Cette tendance rejoint les conclusions récentes formulées dans d'autres travaux, en particulier à Bogota (Dureau et al., 2014), qui soulignent le rôle de réception des migrants que jouent désormais les municipes des périphéries métropolitaines. C'est donc dans ce contexte péricentral que nous allons tenter de rendre compte de la manière dont des migrants d'origine rurale ont pu accéder à l'emploi et au logement au cours de ces dernières années.

\section{Insertion des migrants dans le marché du travail}

19 Appréhender la manière dont les migrants se sont intégrés au marché du travail métropolitain nécessite de considérer plusieurs dimensions: leur position professionnelle au moment de l'enquête, leur trajectoire depuis leur installation en ville, les modalités d'accès à l'emploi et de maintien de l'activité professionnelle, en particulier pour les femmes. Le croisement des sources mobilisées ici autorise cette approche pluri-dimensionnelle. Tout d'abord, les caractéristiques des migrants originaires du Oaxaca et résidant dans le municipe de Naucalpan sont comparées de manière systématique à celles de deux populations de référence : l'ensemble de la population migrante (hors migration métropolitaine) et l'ensemble de la population résidente dans le municipe. Ensuite, les récits de vie permettent de reconstituer quelques trajectoires individuelles du Oaxaca à Mexico - l'enquête ayant ponctuellement été ouverte à des trajectoires depuis les États de Puebla et de Veracruz (cf. note 6).

\section{Positions sur le marché du travail : cadrage à l'échelon du municipe de Naucalpan}

Afin d'intégrer d'éventuels effets de composition dans l'interprétation des résultats, signalons à grands traits les caractéristiques démographiques des populations observées. La population des migrants récents en provenance du Oaxaca et résidente à Naucalpan est jeune: la moitié a moins de 22 ans; au contraire la population des migrants anciens est plus âgée : la moitié a plus de 37 ans - tandis que l'âge médian de l'ensemble de la population résidente est de 28 ans. Les migrants récents sont aussi plus souvent des femmes (+ de $60 \%$ ) que les migrants anciens $(53,4 \%)$ et que l'ensemble de la population résidente à Naucalpan (51,4\%).

D'une manière générale, la ventilation des actif ${ }^{15}$ par position professionnelle est assez proche, à Naucalpan, de celle observée dans l'ensemble de la ZMVM : près de $68 \%$ sont employés ou ouvriers, $20 \%$ travaillent pour leur propre compte, $3 \%$ sont des patrons et $4 \%$ enfin se déclarent ayudante, c'est-à-dire qu'ils sont payés à la tâche ${ }^{16}$. La proportion 
des employés et ouvriers est nettement surreprésentée dans le groupe des migrants récents en provenance du Oaxaca, non seulement relativement à l'ensemble des actifs résidents, mais également à l'ensemble des migrants récents (illustration 8 ) : au total, elle concerne plus de 9 actifs sur 10 - et quasiment la totalité des femmes ${ }^{17}$.

Illustration 8 - Caractéristiques des migrants récents en provenance du Oaxaca, résidant à Naucalpan en 2010

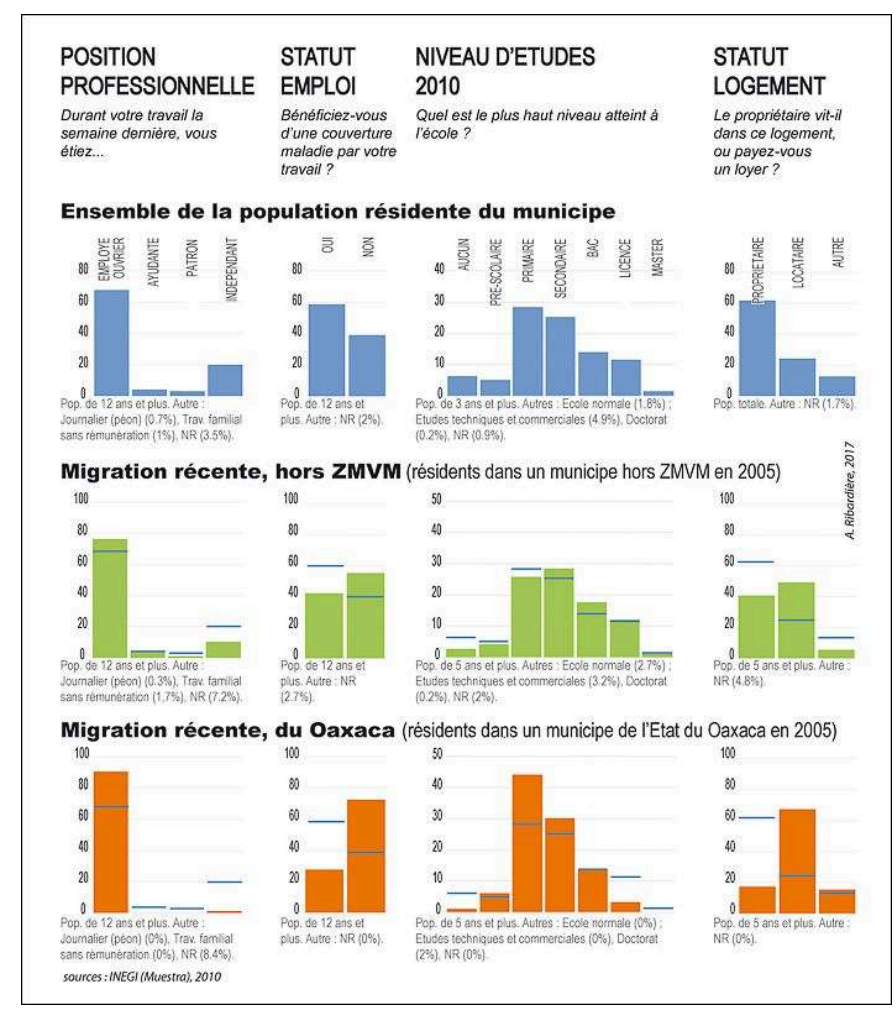

Une autre spécificité des migrants récents du Oaxaca tient au statut de l'emploi. Faire la différence entre le secteur formel et informel est souvent malaisé et plus encore, une telle lecture duale du marché du travail peut ne pas s'avérer pertinente en raison de l'hétérogénéité du secteur informel lui-même (Lautier, 2004). Nous retenons ici une dimension essentielle d'une activité professionnelle déclarée, au moins en partie: l'accès à une couverture maladie ${ }^{18}$. Cette dimension est d'autant plus importante que la famille du salarié bénéficie toute entière de cette couverture. En 2010, seuls $27,5 \%$ des actifs nouvellement venus du Oaxaca occupent un emploi assorti d'une telle couverture : cette proportion apparaît faible non seulement au regard de celle qui caractérise l'ensemble de la population résidente à Naucalpan (59\%), mais également au regard de celle qui caractérise l'ensemble des migrants récents (55,6\%). Ces actifs sont avant tout des hommes ${ }^{19}$.

Le poids de l'emploi non déclaré diminue avec l'ancienneté de l'installation, l'écart entre les taux de couverture des hommes et des femmes également ${ }^{20}$, mais il n'en reste pas moins que pour près de la moitié de l'ensemble des actifs originaires du Oaxaca, l'activité professionnelle ne s'accompagne pas d'un accès à une couverture maladie (illustration 9). 
Illustration 9 - Caractéristiques de la population née dans le Oaxaca et résidente à Naucalpan en 2010

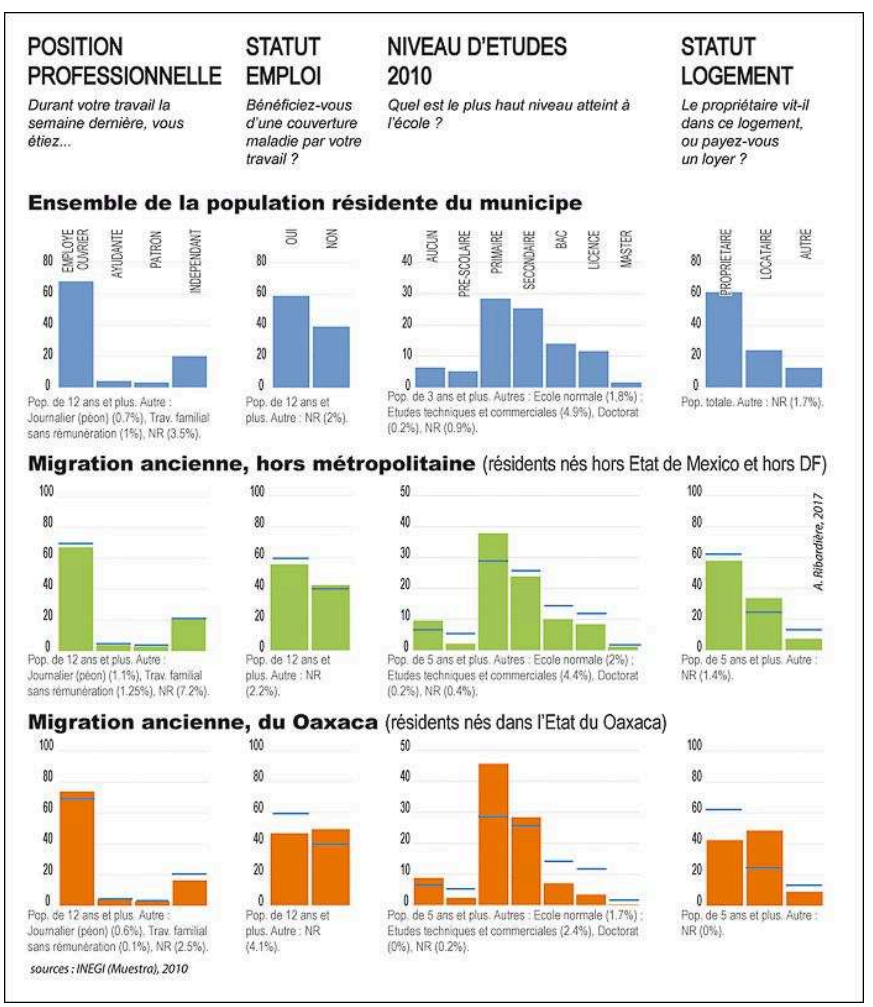

24 Autre trait caractéristique des migrants oaxaqueños : ils apparaissent moins qualifiés que les deux populations de référence, à savoir l'ensemble de la population résidente d'une part et l'ensemble de la population migrante, d'autre part. En particulier, la part des diplômés du supérieur (niveau Licence) est inférieure à $5 \%$ parmi les migrants récents (illustration 8 ). Toutefois, la proportion des personnes ayant achevé un cycle de preparatoria ou de bachillerato (équivalents du baccalauréat) parmi les nouveaux venus est équivalente à celle observée dans l'ensemble de la population résidente. C'est donc dans la surreprésentation du niveau primaire et la sous-représentation du niveau supérieur que se situe la spécificité de la population en provenance du Oaxaca. Ce niveau de qualification médian mérite d'autant plus d'être souligné qu'il distingue les migrants récents (plus jeunes) des migrants plus anciens (plus âgés), pour lesquels l'écart avec l'ensemble de la population résidente est plus important (illustration 9).

Un dernier élément issu de l'exploitation du recensement de 2010 mérite d'être souligné : les écarts observés entre les profils de l'ensemble de la population et les profils des migrants oaxaqueños sont plus accusés à Naucalpan qu'à l'échelle de la métropole tout entière. En effet, si l'on considère l'ensemble des migrants du Oaxaca installés dans la ZMVM depuis 2005 (et non seulement ceux installés à Naucalpan), l'accès à une couverture sociale apparaît alors plus fréquent (+ 4,2 points), la part des titulaires d'une Licence s'avère également plus importante $(+6,8$ points). Ces écarts tendraient à signaler un effet de contexte propre à Naucalpan, mêlant ressources du lieu et ressources du réseau migratoire, qu'il conviendrait de mettre en relation avec les caractéristiques de la population migrante ${ }^{21}$. 


\section{Trajectoires professionnelles}

(

$\mathrm{du}$ travail qui associe emplois informels ou du moins non assortis d'une couverture sociale (emplois domestiques, petit commerce), et emplois relevant sans ambiguïté du secteur formel (postes à l'usine ou dans la vente). Des circulations existent entre les différents segments du marché du travail, qui dessinent dans certains cas des trajectoires ascendantes. Ces trajectoires ascendantes témoignent de ressources propres aux personnes rencontrées, valorisables sur le marché du travail : leur niveau d'études. La figure du migrant rural, n'ayant pas terminé son cycle primaire, existe toujours en 2015 : ces femmes sont alors employées comme domestique, ces hommes tentent de gagner leur vie comme maçon ou militaire. Mais ce n'est pas l'unique figure. Une partie des personnes rencontrées ont bénéficié des politiques éducatives et de 
l'élévation générale du niveau de diplôme, même si de fortes disparités socio-spatiales subsistent (Didou Aupetit, 2011 ; Pérez-Baleón, 2012). Ainsi, d'autres migrants ont non seulement achevé leur cycle primaire, mais sont allés jusqu'à la prepa : la migration vers la métropole constituerait alors un moyen de valoriser leur bagage scolaire, en ayant accès à un marché de l'emploi dans lequel il est monnayable. C'est selon cette hypothèse que l'on peut par exemple interpréter la trajectoire de David : venu à Mexico de Puebla après avoir terminé sa prepa en 2011, il a travaillé quelques mois dans un magasin de chaussures, avant d'être embauché comme vendeur dans un groupe de supérettes il y a trois ans. Il a progressé au sein du groupe, d'abord responsable d'équipe puis, au moment de l'entretien, gérant de la boutique. La trajectoire de Paola constitue un autre exemple : après avoir terminé sa secundaria, elle est venue du Oaxaca voilà une dizaine d'années encouragée par sa tante, esthéticienne à Loma Colorada. Paola a commencé à travailler comme employée domestique et a ensuite suivi une formation d'esthéticienne à San Bartolo.

\section{L'accès au marché du travail}

31 La rencontre entre un migrant, ses ressources en termes de compétences et de niveau d'études et un emploi ne se fait pas sans intermédiaire. Les enquêtes ont montré combien le réseau social constituait un relais essentiel entre le migrant et le marché du travail. La nature de ce lien varie toutefois selon le segment du marché du travail.

Dans les exemples que nous avons rencontrés, le premier placement des domestiques s'effectue toujours par recommandation, via une cousine, une sœur ou une connaissance elle-même domestique. Ensuite, la trajectoire s'autonomise et l'employée explique qu'elle a cherché une nouvelle place par elle-même - même si le système des recommandations est toujours valable.

L'emploi dans une entreprise familiale autorise également la recommandation directe du migrant par le réseau social ou familial. C'est le cas de Carlos, qui travaille dans le garage où est également embauché son père, tandis que ses deux frères travaillent dans deux garages voisins. C'est aussi le cas de Raúl : un de ses frères l'invite à rejoindre un premier groupe de marimba, il monte ensuite son propre groupe, dans lequel il fait entrer son fils et un autre de ses frères.

En revanche, les connaissances et parents des migrants n'ont pas les moyens de placer les nouveaux venus dans l'usine où ils travaillent. Le réseau joue toutefois un rôle essentiel quant à l'accès à l'information: où se trouvent les usines, comment se présenter, comment travailler. Lorena nous expliquait ainsi qu'en arrivant en ville, «on ne savait pas travailler, on ne savait pas se comporter » - non sans humour, elle accompagne ses mots en minant un comportement « convenable ", les mains placées bien parallèles sur les genoux resserrés.

Ainsi, les personnes que nous avons rencontrées n'ont pas témoigné de difficultés particulières à trouver un emploi. Le réseau social et familial a jouté son rôle d'interface, ne serait-ce que dans l'appréhension du marché du travail. Qu'il soit formel ou informel, le travail ne suffit pas toujours à protéger de la pauvreté, toutefois un bagage scolaire équivalent à la prepa, voire à la secundaria, constitue une ressource certaine, reconnue sur le marché du travail et autorise alors des trajectoires ascendantes. 

assez faible, sauf dans le cas des trajectoires ascendantes qui viennent d'être citées
d'une part, ou lorsque cet investissement prend sens à l'échelon d'un projet familial -
c'est le cas des travailleurs indépendants - d'autre part.

\section{La participation des femmes au marché du travail} activité professionnelle. Cette participation au marché du travail est cohérente avec le projet migratoire lui-même et interroge d'autant plus les interruptions de la vie active : au moment de l'enquête, 7 personnes avaient cessé leur activité. En dehors du cas de Cesar, 74 ans, retraité, ces personnes sont des femmes qui expliquent l'arrêt de leur activité par l'arrivée de leurs enfants.

Ces choix renvoient, pour une part, à l'hypothèse d'une insertion professionnelle moins solide, comme en témoignerait le plus faible accès aux emplois formels selon les données de la muestra exploitées plus haut ; ils renvoient également à la place accordée au travail féminin au sein des ménages, telle qu'elle a été étudiée au Mexique de manière très fine par B. Garcia et O. Olivera dans les années 1990 (Garcia et Olivera, 1994). Ces auteures ont montré comment, en particulier dans les catégories populaires, la relation des femmes à leur travail se construisait relativement aux nécessités et aux projets familiaux, et non relativement à un projet personnel. Dans ce contexte, c'est l'éducation des enfants qui va aussi bien légitimer l'arrêt du travail, que la nécessité de travailler. La question de la participation au marché du travail se complique encore pour les migrantes que nous avons rencontrées : le projet migratoire repose en grande partie sur l'accès à l'emploi, mais elles ne peuvent compter sur l'aide de leurs parents pour s'occuper de leurs enfants en bas âge. La résolution de cette question donne alors lieu à des arrangements spécifiques. L'enquête réalisée durant l'été 2015 nous a permis d'en identifier quelques-uns.

Le schéma le plus classique est celui de l'arrêt du travail à la naissance des enfants. C'est la trajectoire de Paola, qui avait commencé sa vie professionnelle comme domestique avant de passer un diplôme d'esthéticienne. Elle a exercé pendant un an son activité auprès de sa tante, pour arrêter lorsque sa première fille est née. Aujourd'hui, elle fait du ménage deux après-midi par semaine (elle amène ses filles sur son lieu de travail) et elle projette de reprendre son travail d'esthéticienne lorsque ses filles pourront rester seules. Ce retour à l'emploi n'est pas systématiquement évoqué. La mise en ménage et la maternité sont aussi apparues comme liées à la sortie d'une vie professionnelle difficile. C'est le cas de Lupita, arrivée à Mexico en 1998 à l'âge de 15 ans seulement. Elle a trouvé sa première place comme domestique par l'intermédiaire de ses frères aînés qui l'avaient accueillie. Elle a cessé de travailler lorsqu'elle s'est mariée et s'occupe aujourd'hui de ses trois enfants.

Un autre schéma classique a été observé chez les enfants de migrants, dans lequel les grands-parents assurent la garde des enfants. Cesar, retraité, a ainsi été rencontré à l'école Anahuac: c'est lui qui s'occupe des enfants de sa fille tandis qu'elle travaille, arrangement qui illustre concrètement le rôle des proximités résidentielles familiales dans les stratégies d'accès et de maintien dans le marché du travail.

41 Toutefois, cette ressource est rarement mobilisable pour les migrants et la garde des enfants peut alors donner lieu à des arbitrages au sein de la fratrie. Florinda s'est ainsi 
occupée des trois enfants de sa sœur aînée. Originaires du Veracruz, les deux sœurs ont migré à Mexico ensemble. Florinda a rapidement trouvé un travail dans une usine comme ouvrière à la chaîne. Ayant terminé sa prépa, elle reconnait elle-même qu'elle aurait pu prétendre à une progression au sein de l'entreprise, mais elle se justifie en expliquant qu'elle ne souhaitait pas avoir de responsabilités. À la naissance du deuxième enfant de sa sœur, elle a interrompu son travail à l'usine pour s'occuper de ses neveux et nièces, tandis que sa sœur, titulaire d'une Licence d'anglais, gère une petite entreprise de coursiers. Aujourd'hui, Florinda tient un commerce de glaces dans une rue passante de Loma Colorada, petite affaire familiale qu'elle gère avec sa sœur. Un arrangement semblable était en projet entre Lorena et sa sœur enceinte au moment des entretiens : Lorena envisageait de garder le bébé de sa sœur, pour lui permettre de garder son travail d'employée.

Enfin, certaines femmes font le choix de conserver leur activité professionnelle coûte que coûte, parce que le double salaire est indispensable à la réalisation du projet familial. Maria a quitté le village de Santo Domingo Petapa en 1993. Comme son mari, elle a travaillé à l'usine quasiment toute sa vie : elle s'est arrêtée uniquement lorsque ses enfants étaient à la maternelle, trop jeunes pour rester seuls à la maison après l'école. Les temps n'ont pas toujours été faciles, mais ses enfants ont pu faire des études: sa fille aînée, titulaire d'une Licence, est comptable, sa cadette a également achevé sa prépa et travaille aujourd'hui dans un centre d'appel tandis que son dernier fils, 17 ans, est encore au lycée. Autrement dit, le double salaire du couple a permis aux enfants de ne pas entrer dans la vie professionnelle trop tôt et ce faisant, de se doter d'un niveau de diplôme qui leur ouvre des perspectives professionnelles plus larges que celles de leurs parents.

L'exemple de ces trajectoires de migrantes permet de mettre en évidence deux éléments en termes de ressources urbaines. Le premier consiste en l'insuffisance des modes de garde proposés dans la ville de Mexico, au moins dans le champ professionnel étudié où les ouvrières font les « trois-huit » et où les employées ne sont pas maitresses de leurs horaires de travail. L'arrêt du travail salarié ne signifie pas l'arrêt de toute activité professionnelle, mais il s'agit alors d'une activité secondaire, qui n'est pas citée comme telle. Le deuxième consiste en le rôle des arrangements familiaux pour pallier l'insuffisance des institutions. Certes, ces arrangements ne sont pas le propre des classes populaires ${ }^{26}$, toutefois dans le cas des familles migrantes rencontrées, cette solidarité a un coût qui est supporté inégalement entre les membres de la famille : les arbitrages semblent se faire relativement aux ressources des un(e)s et des autres sur le marché du travail - en termes de compétences professionnelles en particulier.

Le projet migratoire apparaît bel et bien réalisé pour ces migrants du Oaxaca, motivés par une absence de perspectives en termes d'emploi dans le lieu de départ. Les trajectoires professionnelles apparaissent d'autant plus diversifiées que les migrants bénéficient de la hausse générale du niveau d'éducation au Mexique et/ou qu'elles s'inscrivent dans un réseau qui autorise des arrangements facilitant la réalisation d'un projet professionnel qui fait sens au niveau familial. Cette dimension familiale de l'insertion urbaine transparaît également dans les modalités d'accès à une autre ressource essentielle : le logement. 


\section{L'accès au logement} ou dans une colonie populaire voisine. Cet ancrage périphérique n'exclut pas les changements de résidence, ni la diversité des statuts des logements. Un certain nombre de travaux ont effectivement montré combien le lien entre la forme de production dominante des colonies populaires - l'auto-construction - et le statut de propriétaire était réducteur : dès les années 1980, R. Coulomb insiste sur l'importance du parc locatif dans ces contextes résidentiels populaires, qu'il met en relation avec la consolidation et la densification progressive du tissu urbain (Coulomb, 1988). Plus récemment, les travaux de J.-F. Valette ont également mis en évidence le poids du marché locatif dans les colonies populaires, sous la forme de la mise en location d'une partie du logement du propriétaire, de l'ensemble de la maison ou encore, sous la forme de petits immeubles de rapport (Valette, 2014). Cette diversité du marché du logement, en accession ou en location, ouvre aux nouveaux arrivants des modalités, voire des stratégies d'accès au logement différenciées.

\section{Statut du logement : le poids de la location}

L'exploitation de la muestra de l'INEGI permet de mettre en évidence l'importance du recours à la location chez les migrants récents, supérieure à l'ensemble de la population résidente, quelle que soit la provenance de la migration. Ce fait établi à l'échelle de la zone métropolitaine s'avère plus prégnant encore à l'échelle de Naucalpan ${ }^{27}$ : parmi l'ensemble des personnes installées depuis moins de 5 ans dans le municipe, $44 \%$ résident dans un logement en location, contre à peine $24 \%$ du total de la population résidente. Les migrants oaxaqueños s'inscrivent dans cette tendance de manière toutefois nettement plus accentuée, puisque le recours à la location constitue l'option la plus commune : elle concerne près de 7 personnes sur 10 (illustration 8 ). Ce trait s'atténue avec la durée de l'installation, mais persiste néanmoins: près d'une personne sur deux née dans le Oaxaca réside dans un logement en location en 2010 (illustration 9). Enfin, l'âge médian des propriétaires est significativement élevé : la moitié des propriétaires nés dans le Oaxaca a plus de 50 ans - contre 32 ans seulement si on considère l'ensemble de la population du municipe. Ce serait donc seulement en fin de parcours résidentiel que l'accession à la propriété serait possible.

Les trajectoires résidentielles des personnes rencontrées illustrent de manière exemplaire ces quelques valeurs chiffrées.

\section{Trajectoires résidentielles : entre contraintes et stratégies familiales}

L'arrivée dans la zone métropolitaine mobilise de manière expresse le réseau social du migrant, sur lequel repose l'hébergement des premiers jours. La durée de l'hébergement est d'autant plus courte que les hôtes se connaissent peu; elle peut être 
un peu plus longue lorsque les hôtes sont frères et sœurs par exemple. Après les premières semaines, une fois le premier emploi trouvé, le premier salaire touché, le migrant se tourne vers le marché locatif et cherche à louer un cuarto (logement d'une seule pièce) dans une vecindad ${ }^{28}$, un immeuble ou une maison. La recherche s'effectue à partir des annonces plaquées directement sur le logement ou plus encore, en se renseignant dans le quartier lui-même, cette voie d'accès au marché apparaissant tant cause que conséquence d'une recherche très localisée. Le marché locatif se révèle largement ouvert : il n'est pas besoin de recommandation pour y entrer. Au contraire, l'achat d'un terrain, première étape vers l'accession à la propriétée ${ }^{29}$, nécessite l'intervention du réseau familial ou social.

Parmi les 20 ménages rencontrés, 11 sont en location, 6 sont propriétaires et 3 sont hébergés dans la maison familiale - il s'agit alors d'enfants de migrants. Parmi ceux qui sont en location ou hébergés, 3 sont en cours d'accession à la propriété. La surreprésentation des locataires permet de prendre la mesure de la ressource que peut constituer le marché locatif dans cette ville de propriétaires, pour des familles dont la trajectoire est en construction. La location peut constituer une étape vers l'accession, mais elle peut aussi participer d'autres stratégies familiales.

51 Suivant le schéma le plus classique, la location est en effet une étape nécessaire avant le statut de propriétaires - le ménage loue, au prix le plus bas possible, avant l'achat du terrain et pendant la construction. C'est la trajectoire de Maria, dont nous avons précédemment retracé la trajectoire professionnelle dans les usines de la zone industrielle de Naucalpan et alentour. À son arrivée à Mexico en 1993, avec son mari et sa fille aînée alors âgée de 2 ans, elle a été hébergée durant les deux premiers mois par un oncle résidant à Loma Colorada, le même qui avait quelques années auparavant accueilli sa sœur. Elle a ensuite loué pendant 3 ans un cuarto dans une vecindad de la colonie, où est née sa deuxième fille. Elle rappelle le grand dénuement dans lequel le couple a vécu (absence d'ustensiles de cuisine par exemple) : il s'agissait d'économiser autant qu'il était possible de le faire. Sur les indications d'un collègue d'usine de son mari, également originaire du Oaxaca, ils parviennent à acheter un terrain dans la colonie voisine de Chimalpa et vivent pendant deux mois dans une cabane en bois, le temps que la première pièce soit construite sur le terrain. La maison a été progressivement agrandie : elle compte aujourd'hui 2 étages, plus un garage ouvert audessus duquel est installée la cuisine. Une telle trajectoire résidentielle a conduit la famille de Maria aux franges de l'agglomération, là où le foncier était le plus accessible, redessinant ainsi le modèle proposé par Turner à l'échelle du municipe de Naucalpan.

Ce schéma, qui a été beaucoup celui de la génération des parents des personnes rencontrées, semble difficile à mettre en œuvre aujourd'hui. Parmi les personnes en cours d'accession à la propriété, seul Enrique, fils de migrant, instituteur à l'école Anahuac, a pu acheter un terrain pour y faire construire sa maison. Dans les autres cas, le terrain a été donné au couple par les beaux-parents, résidents de la zone métropolitaine - ce terrain pouvant provenir d'une division d'une parcelle déjà construite, alimentant le processus de densification du tissu urbain des colonies populaires. Quoi qu'il en soit, ce sont les ressources liées à l'ancrage de la belle-famille, et non les ressources du migrant, qui sont ici mobilisées.

Ainsi, le marché locatif reste une ressource locale absolument nécessaire pour ceux qui n'ont pas accès au foncier. Les trajectoires résidentielles s'effectuent alors au sein du parc locatif des colonies populaires. C'est le cas de Carlos, qui vit avec son père et ses 
deux frères, les quatre étant employés dans un garage au centre de Mexico : ils ont loué un cuarto à Tlalpan (au sud du DF), puis à Naucalpan et actuellement à Ecatepec, où ils ont trouvé plus grand et moins cher qu'à Naucalpan. C'est une connaissance qui leur avait parlé des possibilités de logement à Ecatepec et ils ont trouvé grâce à une annonce, affichée sur le logement lui-même.

\section{Louer pour accéder à la centralité} avons observé les modalités d'insertion dans la société urbaine de migrants d'origine rurale, à travers les formes d'accès à l'emploi et au logement. Depuis les premiers temps de l'arrivée en ville jusqu'au moment de l'enquête, c'est finalement à une échelle le plus souvent locale que ces ressources urbaines ont été mobilisées et éventuellement, progressivement construites, même si des trajectoires en direction de colonies populaires plus éloignées ou encore vers le centre ont aussi été observées.

Cet ancrage local ne signifie pas pour autant immobilité. Des parcours professionnels se sont dessinés, articulant emplois informels, mais aussi formels, parfois dans un sens ascendant. Le marché de l'emploi métropolitain se révèle alors comme le contexte nécessaire à la valorisation du bagage scolaire qu'ont pu se constituer certains représentants des catégories populaires, éloignant ainsi leurs trajectoires des stratégies de subsistance décrites par O. Lewis ou L. Lomnitz au cours des décennies 1960 et 1970. De même, des trajectoires résidentielles diversifiées ont pu être mises en évidence à 
l'échelle même du municipe de Naucalpan. Le modèle classique articulant location et accession à la propriété apparaît aujourd'hui de plus en plus difficile à mettre en œuvre, toutefois le recours au parc locatif ne saurait être interprété uniquement comme une option retenue par défaut. Dans certains cas, il témoigne au contraire d'un arbitrage en faveur de la réalisation d'un projet professionnel qui nécessite investissement financier, mais également, accès à la centralité - centralité qui peut se définir également à l'échelle du municipe. Dans tous les cas, tout autant que le potentiel local en matière d'emplois, l'existence d'un parc locatif dans les colonies populaires apparaît comme un élément essentiel pour comprendre le rôle des zones péricentrales dans l'accueil des migrants. Pour mieux comprendre ce rôle, il conviendrait de creuser l'interprétation des écarts que nous avons mentionnés au sujet de Naucalpan, entre la position professionnelle et résidentielle des migrants originaires du Oaxaca résidantdans le seul municipe, et celle observée pour l'ensemble de la ZMVM. Ces écarts pourraient en effet révéler des différentiels de ressources accessibles entre centre et périphérie, et également entre espaces périphériques, qu'il s'agirait d'identifier et de relier aux capacités des individus à se saisir de ces ressources.

$\mathrm{Au}$ total, l'entrée par les ressources urbaines a permis d'une part, d'enrichir la description d'un contexte résidentiel en considérant, au-delà des seules caractéristiques sociodémographiques de la population résidente, les ressources potentielles du lieu et d'autre part, d'intégrer ce contexte dans la compréhension des trajectoires individuelles. Ainsi, la mise en évidence des ressources du lieu a permis de souligner les capacités inégales des uns et des autres à accéder à ces ressources, capacités déterminées tant par les ressources individuelles, en matière de bagage scolaire notamment, que par les ressources et stratégies du réseau familial. En retour, la diversité des trajectoires professionnelles et résidentielles observées à un échelon local rend bien compte de l'hétérogénéité des colonies populaires de la zone métropolitaine. L'exemple de l'insertion urbaine des migrants du Oaxaca montre combien cette diversité résidentielle et fonctionnelle constitue une ressource pour le développement des individus; cette diversité participe également à expliquer les trajectoires de rattrapage (et non de paupérisation) des colonies populaires relativement au reste de l'agglomération (Ribardière et Valette, 2013) et ce faisant, leur insertion progressive au tissu urbain.

\section{BIBLIOGRAPHY}

Baby-Collin V., Cortes G., Faret L., Sassone S., 2009. Chapitre 6 - Une approche comparée des circulations migratoires latino-américaines : les cas bolivien et mexicain. In Cortes G., Faret L., Les circulations transnationales, Paris, Armand Colin, p. 91-106.

Blanchard S., 2014. Migration féminine et « condition domestique » : de l'apprentissage à la professionnalisation. Revue Tiers Monde, $\mathrm{n}^{\circ}$ 217, p. 147-162.

Connolly P., 1981. Autoconstrucción espontánea ¿solución o problema? Revista INFONAVIT VIVIENDA, nº 42, p. 144-153. 
Cortes G., Pesche D. 2014. Territoire multisitué. L’Espace géographique,nº 42, p. 289-292.

Coulomb R., 1988. Logement locatif et dynamique de l'habitat dans la ville de Mexico. Revue de géographie de Lyon, $\mathrm{n}^{\circ}$ 631, p. 9-25.

Delaunay D., 2010. Mobilités, ségrégations résidentielles et bonus démographique dans la zone métropolitaine de Santiago du Chili. Revue Tiers Monde, n² 201, p. 65-85.

Didou Aupetit, S., 2011. Inégalités scolaires et ethnicisation dans l'enseignement supérieur au Mexique. Autrepart, $\mathrm{n}^{\circ}$ 59, p. 19-35.

Duhau E., Giglia A., 2008. Las reglas del desorden: habitar la metrópoli. México, Siglo XXI, 570 p.

Dureau F., Lévy J.-P., 2010. Villes et mobilités au Nord et au Sud : la construction d'une problématique commune. Autrepart, $\mathrm{n}^{\circ} 41$, p. 135-148.

Dureau F., Lulle T., Souchaud S., Contreras Y., 2014. Mobilités et changement urbain : Bogotá, Santiago et São Paulo. Rennes, Presses universitaires de Rennes, 438 p.

Fassin D., 1996. Exclusion, underclass, marginalidad. Figures contemporaines de la pauvreté urbaine en France, aux États-Unis et en Amérique latine. Revue française de sociologie, $\mathrm{n}^{\circ} 371$, p. 37-75.

García B., Oliveira, O., 1994. Trabajo femenino y vida familiar en México. México, El Colegio de México, $301 \mathrm{p}$.

Lautier B., 2004. L'économie informelle dans le tiers monde. Paris, La Découverte, 128 p.

Lewis O., 1960. The Culture of Poverty in Mexico City-Two Case Studies. The Economic Weekly, vol. 12, n²3- 24-25, p. 965-972.

Lomnitz L. de., 1978. Cómo sobreviven los marginados. México, Siglo Veiuntiuno, 229 p.

Lomnitz L. de, Perez-Lizaur M., 1987. A Mexican elite family, 1820-1980: kinship, class, and culture. Princeton N.J., Princeton University Press, 294 p.

Ludec N., 2002. De la campagne à la ville : les employées domestiques à Mexico. Amérique Latine Histoire et Mémoire. Les Cahiers ALHIM, 4.

Michel A., Prunier D., Faret L., 2011. Familles migrantes et ancrages locaux au Mexique : trajectoires et patrimoines migratoires dans la région de Tehuantepec. Autrepart, $\mathrm{n}^{\circ}$ 57-58, p. 77-94.

Michel A., Ribardière A., 2016. Croissance des petites villes au Mexique : quelles formes d'urbanisation? Territoire en mouvement, Revue de géographie et aménagement [En ligne], 32 | 2016, mis en ligne le 23 novembre 2016, URL : http://tem.revues.org/3909

Pérez-Baleón G.-F., 2012. Análisis por cohorte, género y estrato socioeconómico de la salida de la escuela. Estudios Demográficos y Urbanos, vol. 27, núm. 3(81), p. 699-737.

Pérez E., Santos C., 2013. Tendencias recientes de la migración interna en México. Papeles de población, vol. 1, n 76, p. 53-88.

Quesnel A., 2009. De la communauté territoriale à l'organisation familiale en archipel : la mobilité spatiale des familles rurales en Afrique de l'Ouest et au Mexique. In Dureau F., Hily M.-A., Les mondes de la mobilité, Rennes, Presses Universitaires de Rennes, p. 67-103.

Ribardière A., Valette J.-F., 2013. Évolution de la division sociale de l'espace urbain à Mexico. Approche à partir des structures démographiques et de l'accès au diplôme 1990-2010. Problèmes d'Amérique latine, $\mathrm{n}^{\circ}$ 90, p. 99-126. 
Ribardière A., Valette J.-F., 2014. La pauvreté urbaine à Mexico : une approche de la diversité des colonies populaires. EchoGéo [En ligne], 30 | 2014, mis en ligne le 04 décembre 2014. URL : http:// echogeo.revues.org/14020 ; DOI : 10.4000/echogeo.14020

Schteingart M., 1989. Dinámica poblacional, estructura urbana y producción del espacio habitacional en la zona metropolitana de la ciudad de México. Estudios Demográficos y Urbanos, vol. $43, \mathrm{n}^{\circ} 12$, p. 521-548.

Schteingart M., 2002. La división social del espacio en las ciudades. Perfiles Latinoamericanos, $\mathrm{n}^{\circ} 19$, p. 13-31.

SEDESOL (Secretaria de desarrollo social), CONAPO (Consejo Nacional de población), INEGI (Instituto Nacional de Estadística, Geografía e Informática), 2012. Delimitación de las zonas metropolitanas de México 2010. México.

Turner J., 1968. Housing Priorities, Settlement Patterns, and Urban Development in Modernizing Countries. Journal of the American Institute of Planners, $n^{\circ} 346$, p. 354-363.

Valette J.-F., 2014. Mobilités et ancrages dans les quartiers populaires de la périphérie de Mexico. Une approche de la maturation urbaine. Thèse, Paris, Université Paris 1 Panthéon-Sorbonne, 956 p.

Valette, J.-F., 2016. L'hétérogénéité des colonias populares de la périphérie de Mexico : une approche des micro-divisions sociales dans le processus de maturation urbaine. L'Espace géographique, $\mathrm{n}^{\circ}$ 44, p. 289-306.

Vidal D., 2007. Les bonnes de Rio : emploi domestique et société démocratique au Brésil. Villeneuve d'Ascq, Presses universitaires du Septentrion, $312 \mathrm{p}$.

Viramontes R., Vázquez Y., Ramírez J., 2013. Tendencias de la migración interna en México en el periodo reciente. La situación demográfica de México 2013. CONAPO, p. 83-106.

\section{NOTES}

1. La migration entre zones rurales et villes (hors zones métropolitaines) représente quant à elle encore 5,3\% des flux. Selon les calculs effectués par ces auteurs, $68,4 \%$ des flux sont occasionnés par des migrations de type urbain-urbain, autrement dit entre villes et/ou zones métropolitaines ; 12,3\% par des migrations de type rural-urbain/zones métropolitaines et enfin, 3,6\% par des migrations rural/rural. La migration intra-urbaine n'est pas prise en compte dans ces calculs (Pérez et Santos, 2013).

2. Pour une mise en perspective de l'usage de la notion en Amérique Latine, voir Fassin, 1996.

3. Une quinzaine d'années auparavant, c'était sur les vecindades du centre taudifié qu'Oscar Lewis avait porté son regard pour mettre en exergue les éléments d'une culture de la pauvreté (Lewis, 1960).

4. En 2014, le CONEVAL (Consejo Nacional de Evaluación de la Política de Desarrollo Social) estime la part de la population mexicaine touchée par la pauvreté à 46,2\%. L'État de Oaxaca apparaît le deuxième État le plus pauvre du Mexique, avec $65,2 \%$ de la population concernée - après l'État du Chiapas, avec 76, $2 \%$. Pour information, la part de la population touchée par la pauvreté s'élève à $28,4 \%$ dans le District Fédéral et à $49,6 \%$ dans l'État de Mexico. Les écarts sont encore plus frappants si l'on considère la pauvreté « extrême » : 9,5\% en moyenne dans le pays, 28,3\% dans le Oaxaca, 1,7 \% dans le DF, 7,2 \% dans l'État de Mexico (CONEVAL, 2015).

5. Le terme de colonie populaire renvoie à un habitat majoritairement auto-construit, au statut foncier irrégulier et dépourvu des services urbains de base au moment de sa fondation. 
6. Dans le cadre de ce travail, nous retenons 21 entretiens de type semi-directifs, d'une durée variable - environ 1 heure pour la plupart, certains entretiens ayant toutefois été largement prolongés et répétés. Au total, ces entretiens permettent d'appréhender la trajectoire de 20 ménages. 16 personnes sont les acteurs de leur migration (migrants " actifs »); 2 ont migré enfant avec leurs parents (migrants "passifs»); 4 personnes sont enfants de migrants et nés dans la zone métropolitaine. Parmi les migrants, les dates d'arrivées à Mexico s'étalent entre 1977 et 2013, près de la moitié sont arrivés entre 1990 et 2000. L'âge des personnes rencontrées varie entre 18 et 74 ans, avec une surreprésentation des personnes dans la trentaine et la quarantaine. 16 personnes sont originaires du Oaxaca, 3 du Veracruz et 3 de Puebla. Enfin, 18 entretiens ont été réalisés à Naucalpan, 2 dans le centre du District Fédéral et 1 dans la délégation Iztapalapa, tous en juin 2015. Le lieu principal de l'enquête - la colonie populaire de Loma Colorada - et le mode de recrutement des personnes enquêtées, de proche en proche, induit un échantillon composé de personnes de condition modeste d'une part, et une surreprésentation des femmes dans l'échantillon d'autre part : 16 femmes pour 5 hommes. Les prénoms des personnes enquêtées ont été modifiés.

7. Cet échantillon de la muestra ne décrit pas uniquement une population d'origine rurale : en 2010, parmi les migrants récents en provenance du Oaxaca, près d'un tiers venait de la capitale de l'État - toutefois une proportion encore plus grande, soit 1 personne sur 4, venait d'une ville de moins de 10000 habitants. Pour une présentation de la manière dont le recensement permet d'appréhender les migrations, voir infra, première partie, section 3.

8. Ce travail a été réalisé dans le cadre d'un programme PICS du CNRS « Ressources urbaines et trajectoires familiales dans le Mexique des années 2010 (RESUM) » ainsi que dans le cadre d'une mission longue durée IRD en juin-juillet 2015.

9. La ZMVM (zone métropolitaine de la vallée de Mexico) s'étend sur 3 États : elle comprend les 16 délégations du District Fédéral, 56 municipes de l'État de Mexico et 1 municipe de l'État d'Hidalgo (SEDESOL, CONAPO, INEGI, 2012). Cf. illustration 1.

10. 14 variables du recensement ont été considérées simultanément, permettant de saisir le niveau d'accès à l'éducation et à la santé, les conditions de logement, le niveau d'équipement des ménages et enfin, la représentation des populations « indigènes » (Ribardière et Valette, 2014).

11. Population de 12 ans et plus.

12. Pour une présentation de la manière dont le recensement permet d'appréhender les migrations, voir infra, prochaine section.

13. Désigner la population résidente née en dehors de l'État de Mexico comme «migration ancienne" relève, pour une part, d'une facilité de langage: cette population comprend également les migrants récents (moins de 5 ans) nés dans un autre État que l'État de Mexico. Nous retenons toutefois cette désignation pour assurer la fluidité du texte.

14. Les 3 délégations centrales ont accueilli 14,8\% de la migration 2005-2010 hors DF et hors État de Mexico; les 3 délégations/municipes cités ont accueilli 20,6\% de cette même migration (Muestra, 2010).

15. Population active occupée de 12 ans et plus. La législation autorise le travail des mineurs à partir de 14 ans seulement, toutefois dans les recensements le choix a été fait de conserver le seuil de 12 ans, de manière à rendre compte du travail des enfants.

16. La position d'ayudante est définie comme suit par l'INEGI : «Persona de 12 y más años de edad que (...) trabajó por un sueldo o pago, contratado para realizar tareas de corta duración, solo por unas horas o algunos días de la semana y les pagan en cuanto termina la tarea asignada» (Personne de 12 ans et plus qui travaille pour un salaire ou une paye, engagée pour effectuer des tâches de courte durée, pour quelques heures ou quelques jours seulement et rémunérée aussitôt après la tâche réalisée), (INEGI, 2010).

17. Dans l'échantillon de la muestra de 2010, parmi les migrants récents en provenance du Oaxaca et résidant à Naucalpan, la totalité des femmes sont employées ou ouvrières; la 
proportion est moindre chez les hommes $(82.5 \%)$, elle s'abaisse au profit de la catégorie «non renseignée » $(15.5 \%)$, à peine $2 \%$ des hommes travaillent pour leur propre compte.

18. Les compléments assortis d'un travail salarié sont désignés sous le terme de prestaciones, ils renvoient à la couverture maladie, mais aussi aux congés payés, aux primes, etc. Dans les faits, un emploi peut être assorti d'une partie seulement des prestations prévues par la loi.

19. Dans l'échantillon de la muestra de 2010, parmi les migrants récents en provenance du Oaxaca et résidant à Naucalpan, aucune femme ne bénéficie d'une couverture maladie; la proportion s'élève à plus de la moitié chez les hommes $(55,8 \%)$.

20. Dans l'échantillon de la muestra de 2010, parmi les migrants anciens en provenance du Oaxaca et résidant à Naucalpan, à peine un tiers des femmes bénéficient d'une couverture maladie $(30,8 \%)$; la proportion reste stable chez les hommes (54,2\%).

21. L'analyse exploratoire des caractéristiques des migrants en provenance des États de Puebla et du Veracruz alimente cette hypothèse d'une insertion plus précaire des migrants installés à Naucalpan, relativement à l'ensemble des migrants de la ZMVM : couverture maladie moins diffusée (migrants en provenance de Puebla), part des titulaires d'une Licence moins importante (migrants en provenance de Puebla et du Veracruz).

22. La seule exception est Melissa, une jeune femme de 18 ans, qui a rejoint son mari à Mexico avec son nouveau-né.

23. Deux emplois se distinguent de cet ensemble, celui d'Enrique, enfant de migrants aujourd'hui instituteur à l'école Anahuac et celui d'Ana, la sœur de Lorena, employée dans l'administration.

24. Notons ici que si le lien entre condition migratoire et condition domestique perdure, il ne résume plus à lui seul le secteur d'activité, également investi par les classes populaires originaires de la zone métropolitaine (Ludec, 2002). Pour une analyse sociologique des transformations du secteur, voir Vidal, 2007.

25. Type de xylophone d'origine africaine, fréquent dans les orchestres populaires du Oaxaca et du Chiapas et plus globalement, d'Amérique Latine.

26. L'importance des réseaux familiaux dans les trajectoires des catégories aisées a été en particulier mise en évidence au Mexique par L. Lomnitz dans ses travaux au cours des années 1980 (Lomnitz et Perez-Lizaur, 1987).

27. Près d'un tiers des personnes ayant changé de municipalité de résidence entre 2005 et 2010 renvoie au statut de locataire, contre $1 / 5$ seulement du total des habitants de la ZMVM (Valette, 2014, p. 415)

28. La vecindad est une forme de logement collectif destiné aux couches populaires, qui peut prendre plusieurs formes, mais qui implique des logements de petite taille ( 1 ou 2 pièces) et des parties communes (cour, éventuellement sanitaires), le tout concourant à une grande promiscuité des ménages résidents.

29. Le milieu très modeste dans lequel s'est déroulée l'enquête n'a pas accès à un autre marché en accession (grands ensembles d'intérêt social par exemple).

\section{ABSTRACTS}

This paper aims at studying the process of insertion of migrants from rural origin in Mexico City. The analysis is built around the concept of urban resources. In this way, we want to highlight how site's resources, individual resources and social and familial network's resources are all 
linked together. This analysis is based on one hand on detailed data of the Mexican's census that allow us to identify the working and housing statuses of the migrants from Oaxaca, and on the other hand on an empirical survey carried out in the industrial district of Naucalapan. It emphasizes the importance of sub-central areas in the reception of migrants and in their access to the urban resources.

Cet article rend compte des modes d'insertion de migrants d'origine rurale dans la ville de Mexico, au prisme de la notion de ressources urbaines. Ce faisant, nous cherchons à restituer comment s'articulent les ressources du lieu, les ressources propres à l'individu et les ressources du réseau social et familial. Établie à partir des données détaillées du recensement permettant de cerner les statuts professionnels et résidentiels des migrants en provenance du Oaxaca et d'une enquête empirique menée dans le municipe industriel de Naucalpan, l'étude illustre le rôle des espaces péricentraux dans la réception des migrants et dans leur accès aux ressources urbaines.

\section{INDEX}

Keywords: Urban resources, Mexico, sub-central areas, migration, employment, housing Mots-clés: ressource urbaine, Mexico, péricentre, migration, emploi, logement

Subjects: Sur le Champ - Sur le Terrain

\section{AUTHOR}

\section{ANTONINE RIBARDIÈRE}

Antonine Ribardière, antonine.ribardiere@univ-paris1.fr, est Maître de conférences à l'Université Paris 1-Panthéon Sorbonne. Elle a publié récemment :

- Ribardière A., Bonerandi-Richard E., Martin M., Merchez L., 2014. La pauvreté dans l'espace européen : grilles de lecture. In Bonerandi-Richard E. et Boulineau E. (coord.), La pauvreté en Europe. Une approche géographique. Presses Universitaires de Rennes, collection Géographie sociale, p. 17-32.

- Ribardière A., 2016. Richesse et pauvreté dans les communes populaires franciliennes : les politiques du logement changent-elles la donne? L'Espace géographique, 1/2016 (Tome 45), p. 61-78.

- Michel A., Ribardière A., 2016. Croissance des petites villes au Mexique. Territoire en mouvement, 32, 23 novembre 2016. http://tem.revues.org/3909 